\title{
A Weighted Filtering Algorithm Based on Virtual Center Of Sub-window
}

\author{
Dehai Shen ${ }^{\mathrm{a}}$, Jian Hou ${ }^{\mathrm{b}}$, Xu E ${ }^{\mathrm{c}}$, Longchang Zhang ${ }^{\mathrm{d}}$, Qi Yan ${ }^{\mathrm{e}}$ \\ College of Information Science and Technology, Bohai University, Jinzhou, Liaoning 121013, China \\ ashendh25@qq.com, bjian_hou@163.com, cexu21@163.com, d22427291@qq.com, \\ e32392392@qq.com
}

Keywords: mixed noise, child window, virtual center, weight.

Abstract. In order to remove the mixed noise in an image, proposed a new weighted filtering algorithm. The algorithm used $5 \times 5$ filtering window, divided the window into sixteen $2 \times 2$ sub-windows, took these median pixel of every sub-window as their virtual center, then calculated the weights of these virtual center points according to the improved IMF method. At last, used these virtual center points and their weights to weight average, the result is used as the filtering output of the filtering window. Experiments indicate that the new algorithm has good filtering performance for images mixed with Gauss noise and impulse noise, the effects are superior to the traditional median filtering algorithm and mean filtering algorithm and IMF algorithm.

\section{Introduction}

Digital images are often influenced in the process of acquisition and transmission, and become blurred. The most common of these noises is Gaussian noise and salt and pepper noise, they often appear at the same time, we called them mixed noise, they will affect the subsequent processing, such as feature extraction, image segmentation and image recognition and so on. Image denoising aims to suppress the influence of various noises, and keeps the detail information of the image at the same time. Mean filter and median filter are two classic filtering algorithms, are often used to remove Gaussian noise and salt and pepper noise respectively. A single method or simple superposition of the two methods is used to remove the mixed noise in an image, the effect is not ideal. Therefore, many scholars have studied and put forward some improved methods [1-4], these methods separate the noise in the image, and then use improved median or mean filtering method to filter out of two kinds of noise, respectively obtained certain noise suppression effect, but they all put regional extreme value point as the impulse noise point, edge information is often miscalculated. The literature [3] use median pixel gray value in the window to alternative center pixel, the noise points in the window are involved in the calculation, the filtering effect is not ideal. Literature [2, 4] use the weighted average method to remove the Gaussian noise, but determination of the weighting coefficient is without considering the location correlation of the window pixels, in the case of relatively high concentrations of Gaussian noise, their filtering effects are poor. In this paper, a kind of weighted filtering algorithm based on virtual center child window is proposed, the algorithm draws lessons from weighted thought of IMF algorithm in literature [5], not only suppresses the different concentrations of mixed noise effectively, but also protects image edge details.

\section{IMF filtering algorithm}

IMF algorithm is a weighted method which has the thought of median filter and means filter at the same time, the algorithm uses adaptive method to calculate weights, and uses weighted average to smooth noise. 
The principle of IMF algorithm is to determine median value of all the pixel gray value in the filtering window, and then calculates each pixel of the weighted coefficient of $w_{k}(i, j)$ on the basis of the median value, the formulas are shown in Eq.1

$$
w_{k}(i, j)=\frac{1 /\left(1+\left(f_{k}(i, j)-\operatorname{Median}(\mathrm{W}[f(i, j)])\right)^{2}\right)}{\sum_{k=1}^{\mathrm{N}}\left(1 /\left(1+\left(f_{k}(i, j)-\operatorname{Median}(\mathrm{W}[f(i, j)])\right)^{2}\right)\right)}
$$

In Eq.1, $\mathrm{N}$ is pixel total in filtering window, its corresponding value in $3 \times 3$ filtering window and $5 \times 5$ filtering window is 9 and 25 respectively. Eq. 1 uses the variance between every point gray value with median value in the window to calculate the weight of each pixel, and normalizes the weights. Finally, each pixel in the filtering window and their corresponding weights weight average, the result is used as the output of the filter window. The weighted filter output is shown in Eq.2.

$$
g(i, j)=\sum_{k=1}^{\mathrm{N}} f_{k}(i, j) \cdot w_{k}(i, j)
$$

The IMF algorithm obtains weighting coefficient by using pixels in the filter window and their median value to take differential arithmetic, the algorithm is not restricted by threshold, plays a good effect in inhibiting Gaussian noise.

\section{The algorithm in this paper}

Draw lessons from the weighted thoughts of IMF algorithm, proposed a new filtering algorithm, the algorithm uses the $5 \times 5$ filtering window, divides the window into 16 sub-windows, then takes the median value of all pixels in each sub-window as the virtual center point, uses improved weighted method to calculate the corresponding weight of virtual center point, and weights average, the results are as the filtering output of filtering window. The steps of filtering process as follows:

Dividing sub-windows. Let $\boldsymbol{F}$ is a digital image, point $(i, j)$ is center of filtering window $\mathbf{W}$ (size is $5 \times 5)$, algorithm divides $\mathrm{W}$ into to 16 sub-windows, each size is $2 \times 2$, these sub-windows are neighborhood around center point of filtering window. The sub-windows are shown in Fig.1.

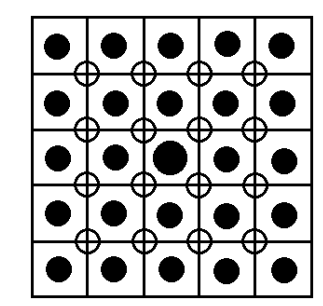

Fig.1 filter window and virtual center points of sub-windows

The 25 solid circles in Fig. 1 represent real pixels in the filtering window W, the largest solid circle in the middle represents the central pixel of the filtering window, and other 16 hollow circles represent 16 virtual central points of the sub-windows.

Calculating gray value of virtual central pixel. In Fig. $1, \mathrm{~W}_{1}-\mathrm{W}_{16}$ represent 16 sub-windows divided, and $Z_{1}-Z_{16}$ represent virtual center pixels of sub-windows for respectively. The algorithm calculates each median of non-salt and pepper noise(the gray is 0 or 255 ) in $\mathrm{W}_{1}-\mathrm{W}_{16}$ firstly, eliminates the influence of salt and pepper noise, each median is as virtual central pixel gray, the algorithm takes advantage of neighborhood correlation principle, and keeps the edge details of the image information well. Set $N$ stores these medians, when density of the salt and pepper noise is 
high, there could be any sub-windows whose pixels are all noise points, so the median is invalid, is not included in $N$. The formula is shown in Eq.3.

$$
N=\left\{x \in \operatorname{Median}\left(W_{k}[F(i, j)]\right) \mid F(i, j) \neq 0 \text { or } F(i, j) \neq 255, k=1,2, \ldots n\right\}
$$

Calculating weights. Finds out average of these medians in set $N$, and then Calculates absolutions $F a_{\mathrm{k}}$ of deference between these medians and their average respectively. The formula is shown in Eq.4. $M_{k}$ presents arbitrary median in the set $N$, Mean $\left(M_{k}\right)$ presents the average. The algorithm uses method in Eq. 5 to calculate the normalized weights of each median.

$$
\begin{aligned}
& F a_{k}=\left|M_{k}-v\right|=\left|\operatorname{Median}\left(Z_{k}[F(i, j)]\right)-\operatorname{Mean}\left(M_{k}\right)\right| \\
& w_{k}(i, j)=\frac{1 /\left(1+\operatorname{Max}\left(F a_{k}, T H\right)\right)}{\sum_{k=1}^{n} 1 /\left(1+\operatorname{Max}\left(F a_{k}, T H\right)\right)}
\end{aligned}
$$

In Eq.5, $w_{k}$ is the weight of each median $\left(M_{1}-M_{k}\right), T H$ is a threshold, its value is the average of all $F a_{\mathrm{k}}$. The algorithm refers to threshold optimization method in literature [5] and improved it, uses absolution of difference between medians in sub-windows and their average to replace the variance. The weight of each median in sub-windows is determined by maximum between absolution $F a_{\mathrm{k}}$ and $T H$. Experiments show that improved method has achieved good practical effect.

Weighting and filtering. The median $\left(M_{1}-M_{k}\right)$ multiple by their corresponding weights and sum up, the result is as the filtering output of central point in filtering window, the method is shown in Eq.6

$$
F^{\prime}(i, j)=\sum_{k=1}^{n} M_{k} \cdot w k
$$

\section{Verification experiment and the effect analysis}

In order to verify the performance of the algorithms, experiments use standard gray image lena with $256 \times 256$ pixels as initial image in Matlab 2010. We mix different density salt \& pepper noise and Gauss noise in initial image each time, use the traditional median filtering algorithm, traditional mean filter algorithm, IMF algorithm and proposed algorithm to filter mixed noise respectively, the results are shown in Fig.2 and Fig.3, the PSNR of these algorithms are shown in table1.

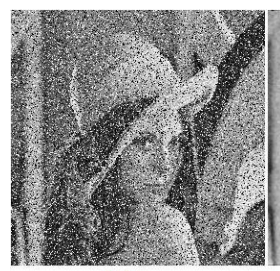

(a)

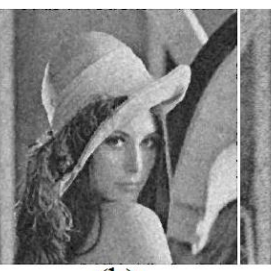

(b)

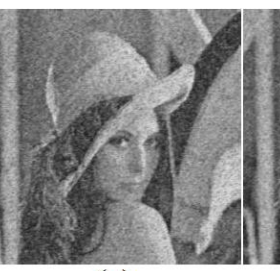

(c)

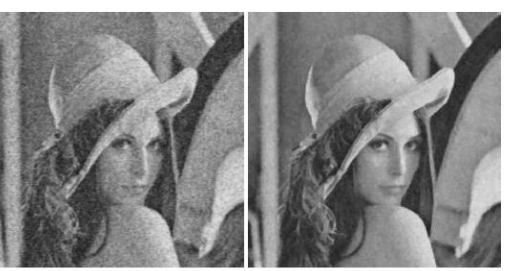

(d) (e)

Fig 2 filtering image with low density Gauss noise and salt \& pepper noise

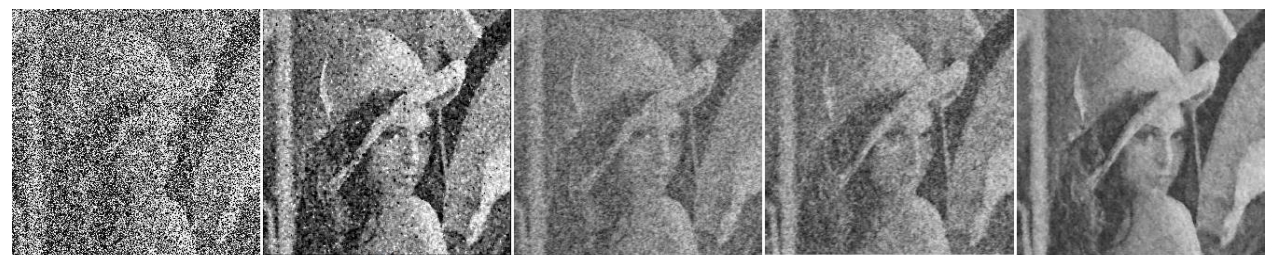

(a)

(b)

(c)

(d)

(e)

Fig 3 filtering image with high density Gauss noise and salt \& pepper noise 
In Fig.2-Fig.3, (a) is mixed noise image; (b) is traditional median filtering image; (c) is traditional mean filtering image; (d) is IMF filtering image;(e) is proposed algorithm image.

In Fig.2, the initial image is mixed with Gauss noise of variance is 0.01 (mean is 0) and salt and pepper noise of 20\%. In Fig.3, the initial image is mixed with Gauss noise of variance is 0.05 (mean is 0 ) and salt and pepper noise of 50\%. From Fig.2 to Fig.3, We can see the filtering image of proposed algorithm is the most clearest, and with increasing of the mixed noise, the denoising performance of the other three algorithms decrease rapidly, but the proposed algorithm have stable denoising ability, the filtering image is clearer than others, and has good details preserving performance.

Table 1 the PSNR comparisons of four algorithms

\begin{tabular}{lccccccc}
\hline Gauss noise & 0.01 & 0.02 & 0.03 & 0.05 & 0.06 & 0.07 & 0.08 \\
Salt \& pepper noise & 0.05 & 0.10 & 0.15 & 0.20 & 0.25 & 0.30 & 0.35 \\
\hline Median & 27.05 & 25.25 & 23.58 & 21.45 & 20.26 & 19.23 & 18.05 \\
Mean & 25.93 & 24.13 & 22.74 & 21.34 & 20.23 & 19.39 & 18.57 \\
IMF & 26.60 & 25.10 & 23.85 & 22.28 & 21.47 & 20.52 & 19.86 \\
NEW & 27.98 & 26.42 & 25.15 & 23.02 & 21.99 & 21.37 & 20.57 \\
\hline
\end{tabular}

Can be seen from table 1, in the case of different mixed noise density, the PSNR of proposed algorithm(NEW) are all higher than other three filtering algorithms. And with the increasing of noise density gradually, the PSNR of proposed algorithm drops slower than the others.

\section{Conclusions}

In order to remove mixed noise, a new weighted filtering algorithm is proposed. The algorithm refered to the weighted idea of the IMF algorithm, constructed a group of virtual center points of sub-windows divided, for these virtual center points, used improved method to weight. Experiments show that the improved algorithm has good denoising effects and details preserving ability.

\section{Acknowledgement}

The project is sponsored by National Nature Science Foundation(61473045), Natural Science Foundation of Liaoning Province(2014020141).

\section{References}

[1] Wan Qian, Xue Ming. Mixed Adaptive Image Denoising Algo rithm Based on Noise Isolation and Wavelet Thresholding. Electronic Sci. \& Tech,vol.24, no.5, p.94-97,2011, (In Chinese).

[2] ZHANG Xu-ming, XU Bin-shi, DONG Shi-yun, et al. Adaptive median-weighted mean hybrid filter. OPTICAL TECHNIQUE,vol.30, no.6, p. 652-655,659,2004, (In Chinese).

[3] YANG Hui, TANG Jian-feng, YANG Li-rong, et al. Study on Removal of Mixed Noise in Images Based on Median Filter and Wiener Filter. Journal of Hengyang Normal University, vol.32, no.6, p.52-55,2011 (In Chinese).

[4] WU Ying, WU Hai-yong. Adaptive mixed filtering method for removing image noise. Computer Engineering and Applications, vol.46, no. 7, p.168-170,2010, (In Chinese).

[5] ZHANG Heng, LEI Zhi-hui, DING Xiao-hua. An Improved Method of Median Filter. Journal of Image and Graphics, vol.9, no.4, p. 408-411, 2004, (In Chinese). 